\title{
Median Based Method for Baseline Wander Removal in Photoplethysmogram Signals
}

\author{
Adewale Emmanuel Awodeyi, Stephen R. Alty and Mohammad Ghavami \\ Faculty of Engineering, Science and the Built Environment (FESBE) \\ Department of Engineering and Design \\ London South Bank University \\ London, United Kingdom \\ \{awodeyia,steve.alty,ghavami@1sbu.ac.uk\}
}

\begin{abstract}
Removal of baseline wander is a crucial step in the signal conditioning stage of photoplethysmography signals. Hence, a method for removing the baseline wander from photoplethysmography based on two-stages of median filtering is proposed in this paper. Recordings from Physionet database are used to validate the proposed method. In this paper, the twostage moving average filtering is also applied to remove baseline wander in photoplethysmography signals for comparison with our novel two-stage median filtering method. Our experiment results show that the performance of two-stage median filtering method is more effective in removing baseline wander from photoplethysmography signals. This median filtering method effectively improves the cross correlation with minimal distortion of the signal of interest. Although the method is proposed for baseline wander in photoplethysmography signals, it can be applied to other biomedical signals as well.
\end{abstract}

Keywords—photoplethysmography, cardiovascular, baseline wander

\section{INTRODUCTION}

Photoplethysmography (PPG) has been used extensively for the detection of cardiovascular disease (CVD). Photoplethysmography (PPG) is a non-invasive optical technique for detecting cardiovascular characteristics from the fingertip while the heart is pumping [1]. It uses an infrared light source to illuminate the finger on one side, and a photo detector placed on the other side measures the small variations in the transmitted or reflected light intensity. However, the additional ultralow-frequency signals introduced due to breathing movements which we refer to as baseline wander (BW) in the acquisition process will result in misdiagnosis. Baseline wander makes interpretation of PPG recordings difficult, so before analyzing the PPG signal, removing the baseline wander is necessary. Breathing frequency components are usually below $1 \mathrm{~Hz}$ [2] and are also different between individuals. Because of their low frequency characteristics, these signals are more difficult to filter out through analog filter and a digital filter. The removal of electrocardiogram (ECG) baseline drift has been reported. However, the removal of photoplethysmogram baseline drift has been reported rarely. In general, there are two parts accompanying algorithm in this research; first one is the method for baseline wander removal using two-stage moving average filter (TSMaF). The second one is the proposed method which is required for baseline wander removal using two-stage median filter (TSMdF). After this brief overview, we discuss research related work on baseline wander in section II. The background of our median filtering will be presented in section III. The methods and experimental results are described in section IV. The correlation co-efficient, scatter plot and root mean square error are used to measure the performance of the proposed method.

\section{RELATED RESEARCH WORK}

Researchers have proposed various techniques and algorithms for baseline wander removal which describe baseline wander from different views with each method having advantages and drawbacks. Xu Lishang et al. [3] introduced wavelet based cascaded adaptive filter using two stages to remove baseline wander, in which a discrete Meyer wavelet filter was used as first stage and cubic spline estimation as the second stage. In addition, Lin Yang et al. [4] introduced cubic spline interpolation using selected starting points of pulse waveform to be the spline interpolation point and cubic spline interpolation according to the stating point to fit the baseline drift. Furthermore, Cao Dianguo et al. [5] introduced a wavelet adaptive filter to remove the baseline drift from pulse waveform using the signals of the high-frequency bands among the wavelet transformed signals as reference inputs and the original pulse waveform added baseline drift as the primary input.

\section{BACKGROUND}

The median filter is currently enjoying greater theoretical attention and practical application. Nonetheless, there are yet relatively few papers published on median filter behaviour. Median filters operate by selecting the middle value of an ascending-ordered sequence of numbers. These numbers are taken from a moving window on the data to be processed. The operation can be used in data processing to reject glitches, align events and to pass step functions as well as enhance discontinuities in the data. The median filter is a data dependent operator which extracts a value from a sequence of numbers also invariant to scaling. That is, if all the numbers in the sequence are scaled or exponentiated (assuming positive numbers), then a given number will occupy the same ordered position before and after scaling. It is also a nonlinear operator, as it does not conform to the definition of linearity. Another interesting property of the median is that the running median filter can be applied along a given trace, or across adjacent traces. While the median filter may be used to filter a trace in time, it is also often used as a spatial process [6]. 


\section{Method And Experiment Results}

As illustrated in Fig. 1, the PPG data extracted from the Physionet MIMIC II database [7] is first filtered and then mean removal before yielding a clean signal called PPG original signal as shown in Fig. 2(a). An artificial baseline wander (BW) is generated by using a combination of sine and cosine waves as displayed in Fig. 2(b). The frequencies of the sine and cosine waves are $0.4 \mathrm{~Hz}$ and $0.2 \mathrm{~Hz}$ respectively, while their corresponding amplitudes are $60 \mathrm{mV}$ and $120 \mathrm{mV}$. Thereby, we have corrupted the PPG original signal with measured amounts of baseline wander to generate the PPG test signal as shown in Fig. 2(c) after mean removal.

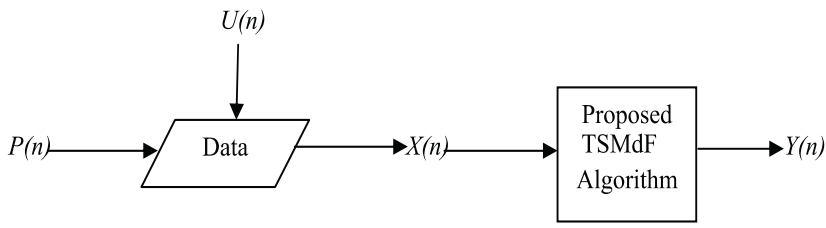

Fig. 1: PPG signal pre-processing.

Where $P(n)$ is a clean PPG original signal contained in $P_{1}$ through $P_{\mathrm{N}}$, and $(P+U) n$ is a corrupted PPG signal. Additionally, $X(n)$ is the output after mean removal (DC offset) and so input to the proposed TSMdF method, whereas $n$ is an index that runs through these values. Therefore, $Y(n)$ is a filtered PPG signal after mean removal. The description of our proposed method is given in algorithm 1 ,

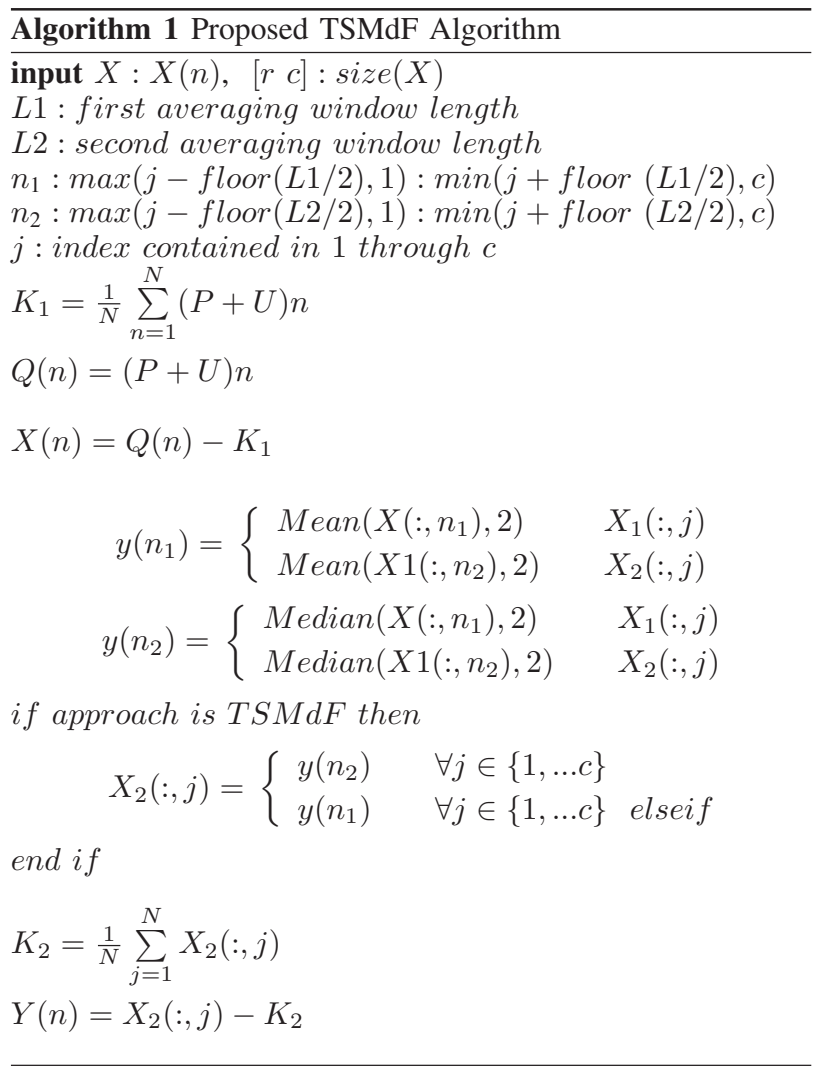

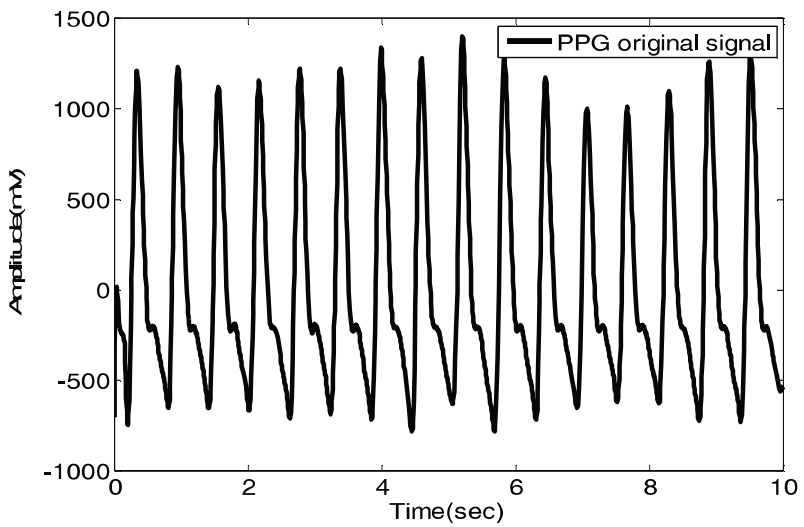

(a)

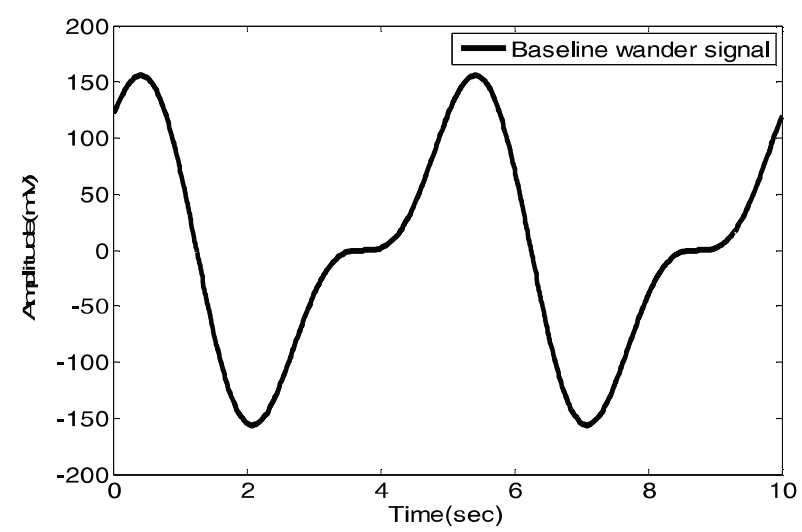

(b)

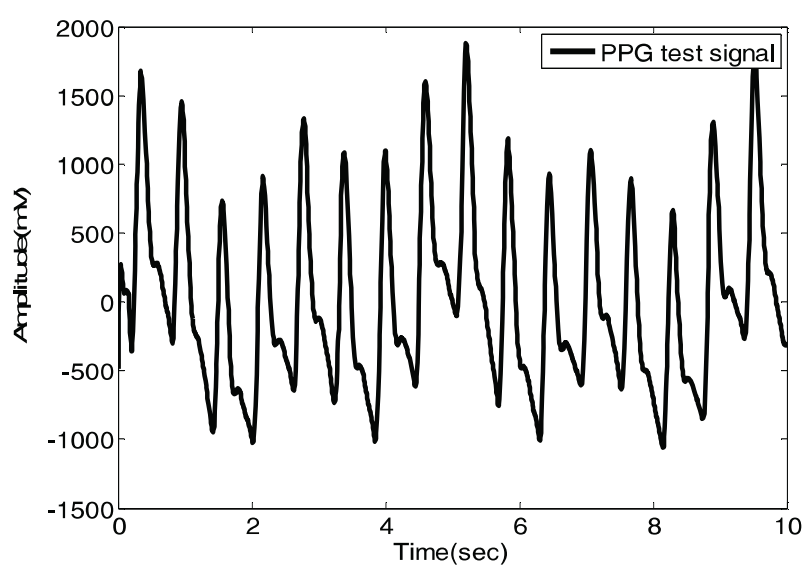

(c)

Fig. 2: Signal types (a) PPG original signal (b) Baseline wander signal (c) PPG test signal.

We applied the two-stage median filter (we will refer to this as TSMdF) method to PPG test signal. The TSMdF have variable window sizes for the removal of BW in samples with an average first stage and second stage of window size set to $0.3^{*}$ sampling rate $\left(f_{\mathrm{s}}\right)$ and $0.6 *$ sampling rate $\left(f_{\mathrm{s}}\right)$ respectively. The PPG signal reconstructed after removing $\mathrm{BW}$ is shown in 
Fig. 3(a). As a comparison, a two-stage moving average filter (TSMaF) is also used to remove BW in the PPG test signal. Fig. 3(b) displays the result of applying the TSMaF to remove the BW. However, careful comparison between Fig. 3(a) and Fig. 3(b) indicate that TSMdf is better at removing BW than TSMaF. In order to compare the results, we evaluate the performance of the correction methods with cross-correlation as expressed in (1).

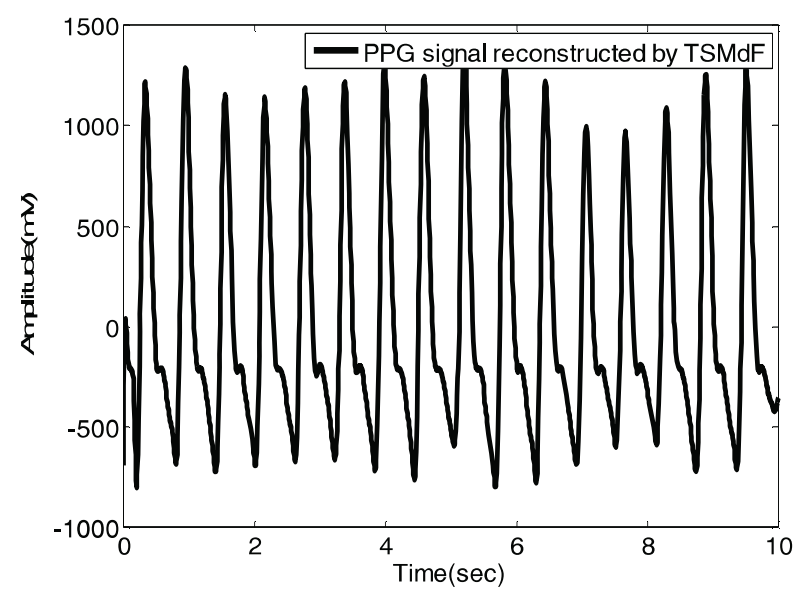

(a)

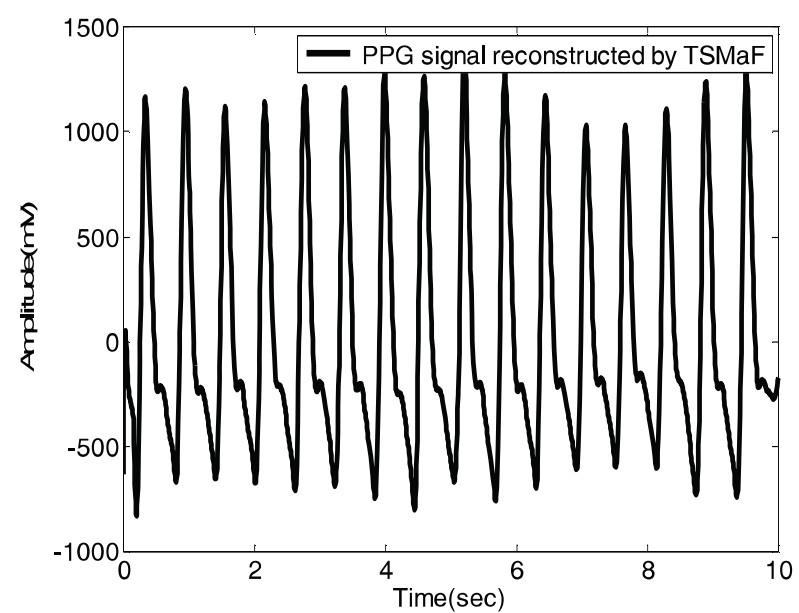

(b)

Fig. 3: Simulation results (a) PPG signal reconstructed by TSMdf (b) PPG signal reconstructed by TSMaF.

$$
R_{x} y=\frac{\sum_{i=1}^{N} X_{i} Y_{i}}{\sqrt{\sum_{i=1}^{N} X_{i}^{2} \sum_{i=1}^{N} Y_{i}^{2}}}
$$

Where $X_{\mathrm{i}}$ is each $X_{1}$ value through $X_{\mathrm{N}}$ of PPG original signal, $Y_{\mathrm{i}}$ is each $Y_{1}$ value through $Y_{\mathrm{N}}$ of the PPG signal reconstructed. The cross correlation $R_{\mathrm{xy}}$ of the PPG original and the PPG signal reconstructed reach 0.9989 for TSMdF correction method while only 0.9975 for TSMaF. The PPG original signal and corresponding PPG signal reconstructed are shown in Fig. 4 and Fig. 5 respectively. The relationship is illustrated graphically using a normalized $X Y$ plot in Matlab. Where two sets of signals are strongly linked together, we say they have a higher positive correlation as in Fig. 6, whereas Fig. 7 shows a noticeably lower positive correlation. The embedding distortion performance is measured by the root mean square error (RMSE). The lower RMSE value the better quality of the signal. RMSE can be defined as the square root of the mean of the square error as expressed in (2). The RMSE comparison results are shown in Fig. 8.

$$
R M S E=\sqrt{\frac{1}{N} \sum_{i=1}^{N}\left(y_{i}-\hat{y}_{i}\right)^{2}}
$$

Where $y_{\mathrm{i}}$ is the value actually observed, $\hat{y}_{\mathrm{i}}$ is the predicted value. The RMSE value for PPG signal reconstructed reach $27.3 \mathrm{mV}$ for $\mathrm{TSMdF}$ whereas $41.8 \mathrm{mV}$ for TSMaF.

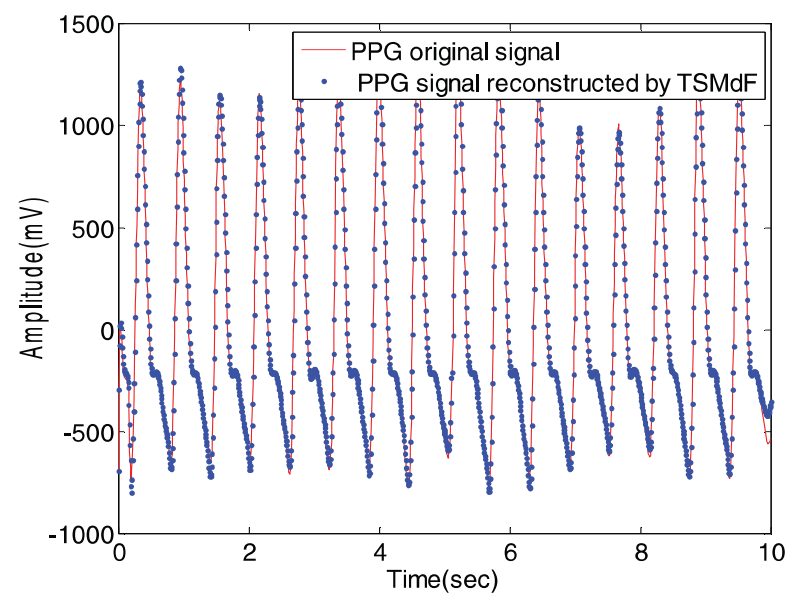

Fig. 4: The solid red line is the PPG original signal and the dotted line is the PPG signal reconstructed by the TSMdF method.

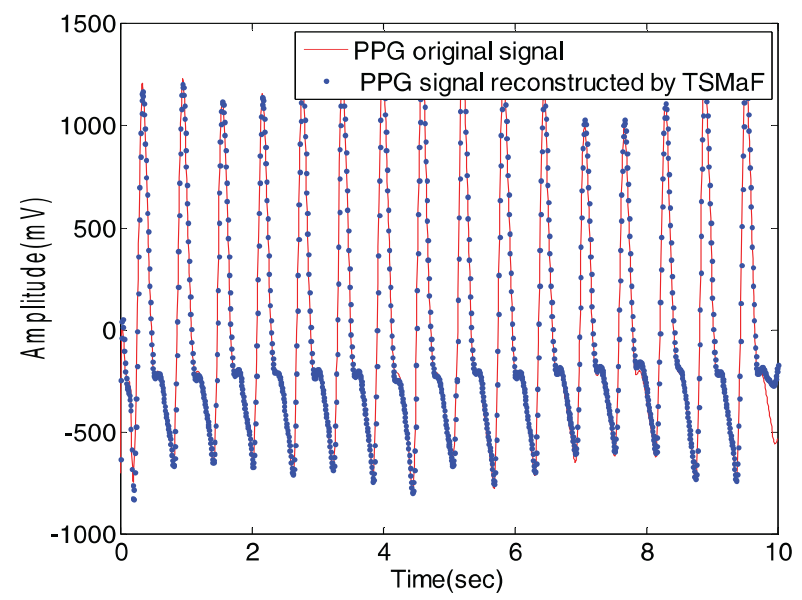

Fig. 5: The solid red line is the PPG original signal and the dotted line is the PPG signal reconstructed by the TSMaF method. 


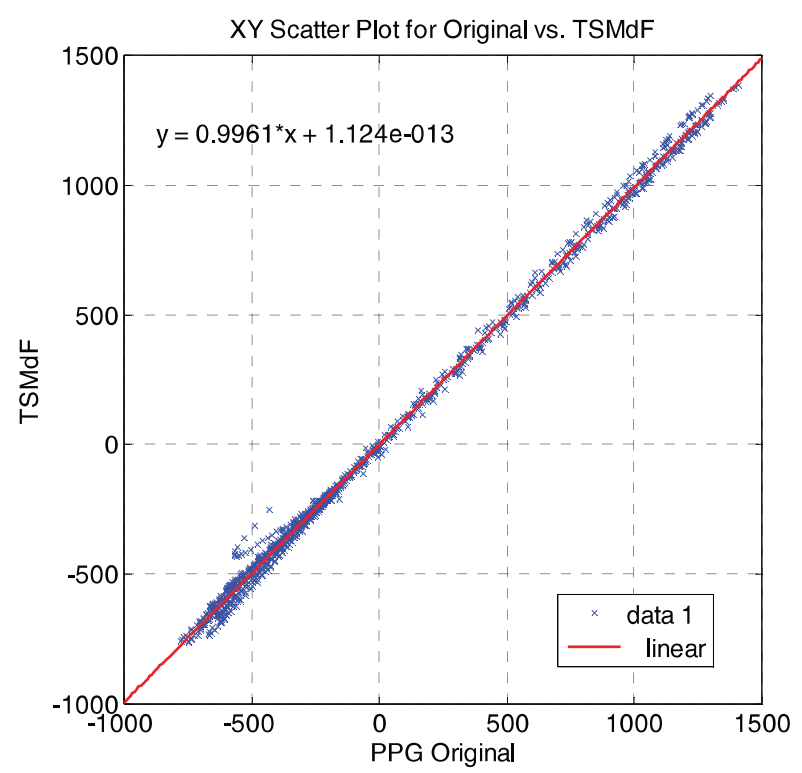

Fig. 6: Scatter plot for TSMdF vs. Original PPG, showing a higher correlation than Figure 7.

\section{CONCLUSiON}

The present paper has used various qualities of measurement. In this paper, the results of experiment measured indicate clearly that our method can give a very satisfactory performance on removal of baseline wander in photoplethysmography signal. Ten extracted photoplethysmography signals from Physionet database were also used to evaluate the performance of the presented method. Work is underway on cascaded filters of photoplethysmography signals to remove other artifacts commonly found in PPG signals: spikes, power line interference and noise. Future work will involve peak detection and heart rate extraction from photoplethysmography signals and data from various patients will be analyzed for healthy and unhealthy classification.

\section{ACCKNOWLEDGMENT}

The first author would like to express his thanks to Biomedical Engineering and Communications (BiMEC) for providing facility to work at London South Bank University and my supervisor for providing moral support and encouragement towards research.

\section{REFERENCES}

[1] J. Allen "Photoplethysmography and its application in clinical physiology measurement", Physiological Measurement, vol. 28, IoP Publishing, 2007.

[2] Zahoor-Uddin, "Baseline Wandering Removal from Human Electrocardiogram Signal using Projection Pursuit Gradient Ascent Algorithm," International Journal of Electrical and Computer Sciences IJECS/IJENS.vol: 9, No: 9, pp11-13, 2011.

[3] Xu Lisheng, Wang Kuanquan, D Zhang, Shi Cheng "Adaptive baseline wander removal in the pulse waveform", Computer-Based Medical Systems, 2002. (CBMS 2002). Proceedings of the 15th IEEE Symposium on, 2002.

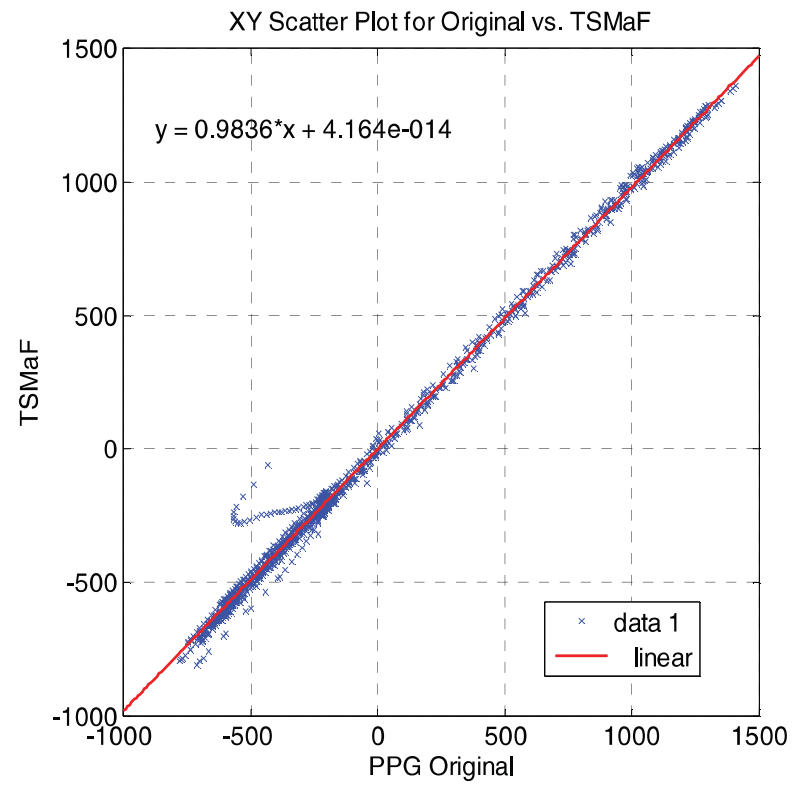

Fig. 7: Scatter plot for TSMaF vs. Original PPG, showing a lower correlation than Figure 6.

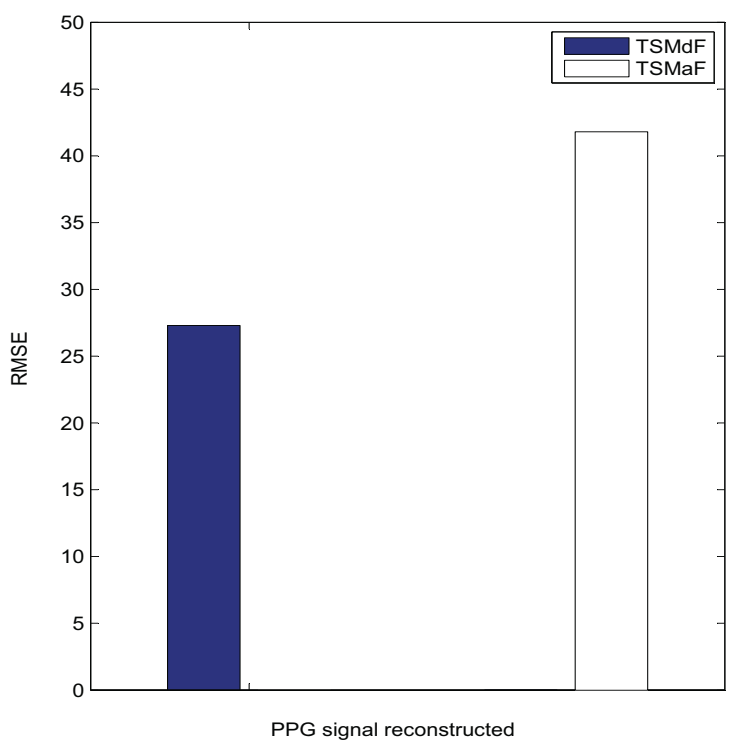

Fig. 8: Comparison between TSMdF and TSMaF.

[4] Lin Yang, Song Zhang, Xiaoyang Li, Yimin Yang "Removal of Pulse Waveform Baseline Drift Using Cubic Spline Interpolation", Bioinformatics and Biomedical Engineering (iCBBE), 2010 4th International Conference on, Page(s): 1 3. 2010.

[5] Dianguo Cao, Liu Liping, Peng Wang, "Removing Baseline Drift in Pulse Waveforms by a Wavelet Adaptive Filter", Bioinformatics and Biomedical Engineering, 2008. ICBBE 2008. The 2nd International Conference on, Page(s): 2135 - 2137, 2008.

[6] Robert R. Stewart, "Review and A New F/K Analogue Design", Median Filtering, vol. 21, pp. 54-63, Dec. 1985.

[7] Physionet Database. Available At http://physionet.org/cgi-bin/atm/ATM, [accessed 02/06/13]. 